\title{
Criminologie
}

\section{Le contrôle informel des gérants de banques et caisses populaires lors d'infractions bancaires}

\section{Reneault Tremblay}

Volume 10, numéro 1, 1977

La criminalité des affaires au Québec

URI : https://id.erudit.org/iderudit/017065ar

DOI : https://doi.org/10.7202/017065ar

Aller au sommaire du numéro

Éditeur(s)

Les Presses de l'Université de Montréal

ISSN

0316-0041 (imprimé)

1492-1367 (numérique)

Découvrir la revue

Citer cet article

Tremblay, R. (1977). Le contrôle informel des gérants de banques et caisses populaires lors d'infractions bancaires. Criminologie, 10(1), 46-68.

https://doi.org/10.7202/017065ar d'utilisation que vous pouvez consulter en ligne. 


\section{LE CONTROLLE INFORMEL DES GERANTS DE BANQUE ET CAISSES POPULAIRES LORS D'INFRACTIONS BANCAIRES}

Reneault Tremblay

Notre société a déjà été étudiée sous plusieurs de ses aspects. Le contrôle social est un de ceux-là. Ce contrôle s'exerce dans un grand nombre de secteurs connus de l'activité humaine et il s'étendra sûrement dans l'avenir à des domaines spécifiques encore partiellement étudiés. Plus rares cependant sont les études portant sur les modes informels de ce contrôle. Notre propos est d'étudier ceux qui entrent en jeu lors de certaines infractions d'ordre économique, en particulier les fraudes bancaires.

Les agissements du fraudeur ont déjà fait l'objet de maintes études, mais ici nous observons l'action de celui qui lui fait obstacle, l'agent de contrôle par excellence, soit le gérant de Banque ou de Caisse populaire. Nous nous attacherons aux facteurs et aux délits qui déclenchent la mise en œuvre des contrôles, et enfin aux résultats de cette stratégie.

Les délits, les contrôles et leurs résultats se situent différemment selon que les institutions bancaires fonctionnent en milieu rural, en milieu semi-urbain ou en milieu urbain. A ce moment-ci de l'histoire des sociétés occidentales et de leur développement, on peut distinguer en disant qu'un contrôle social formel est généralement écrit, que cet écrit est souvent connu sous forme de loi, qu'il a un effet de contrôle sur certains de nos actes sociaux. Le contrôle social informel, par contraste, est celui qui n'est pas écrit ${ }^{1}$. On le reconnaît implicitement plutôt qu'explicitement. Sa force de contrainte sur certains comportements sociaux, bien que provenant de règles tacites, peut cependant être assez forte.

Le contrôle social formel laisse inévitablement échapper beaucoup de crimes. Il est en effet certain que le nombre des délits connus par les tribunaux ne représentent qu'une partie des infractions faites à la loi. Nous faisons donc face au "numerus obscurus» ou «chiffre noir », lequel a été maintes fois étudié. À titre

1. En conséquence, une intervention informelle d'un gérant sera celle qui est verbale (c.-à-d. : téléphone, rencontre personnelle). 
d'exemple, rappelons qu'en 1947 Wallerstain et Wyle ${ }^{2}$ ont prouvé, par un questionnaire de style autoportrait rempli par 1020 hommes et 628 femmes de New York, que 91,9\% d'entre eux avaient commis des offenses passibles d'emprisonnement. La fraude bancaire appartient aussi à ce groupe de délits qui, en presque totalité, n'arrivent pas à l'attention de la Cour, ni même de la police. Qu'arrive-t-il alors de ces cas ? Existe-t-il des moyens efficaces et équitables de déjouer l'infraction ou de la réduire ? Si oui, quels sont-ils? Qui donc s'en sert? Comment? Pourquoi ? En somme, nous aimerions savoir si les prérogatives de la société, si l'intérêt de la victime et celui du délinquant sont mieux servis par le moyen de la conciliation-contrôle informel que par l'intervention forcée de la justice (organe formel), c'est-à-dire par la poursuite, l'inculpation et la condamnation du fraudeur?

Cette recherche ${ }^{3}$ est de style qualitative. La technique d'enquête que nous avons utilisée est essentiellement l'entrevue dirigée centrée. Toutefois, comme nous le verrons ultérieurement, quelques données statistiques seront utiles pour appuyer la première hypothèse. L'échantillon étant restreint, il n'est pas dans notre intention de généraliser les conclusions que nous y apporterons. Nous nous sommes plus attaché à acquérir une meilleure connaissance d'un phénomène peu étudié, que nous n'avons visé à formuler des considérations judicieuses sur la façon de la corriger. Pour comparer les contrôles bancaires informels tels qu'ils fonctionnent en différents milieux, nous avons sélectionné les huit établissements suivants :

En milieu urbain, à Montréal (Ouest) : une Caisse populaire du quartier Côte-des-Neiges (quartier socialement et économiquement à l'aise) ; à Montréal-Est, dans le quartier Hochelaga-Maisonneuve (quartier défavorisé) : une succursale de la Banque provinciale et une de la Banque royale (Succursale communautaire). À Rivière-du-Loup, en milieu semi-urbain : une Banque provinciale, une Caisse populaire et une Banque canadienne nationale. En milieu rural, à Saint-Antonin et à Cacouna (périphérie de Rivière-du-Loup) : une Caisse populaire dans chaque village.

2. Voir Korn, R., McCorkle, I. (1967), Criminology and Penology, New York, Holt, Rinehart \& Winston, p. 4-5.

3. Ce texte est l'abrégé d'un mémoire de maîtrise en criminologie présenté à l'Université de Montréal en 1976. Nous faisons complète abstraction des chapitres de revue de littérature et de l'étude des milieux sociaux. Ainsi, nous ne faisons pas mention de l'apport de Malinowsky, Tonnies, Clinnard, Berger et d'autres à l'aspect informel du contrôle social. 
Nous avons formulé nos hypothèses comme suit :

\section{Première hypothèse}

Nous désirons vérifier si la criminalité officielle et statistique, telle qu'elle ressort du nombre des condamnations, est moins grande (au prorata) en milieu semi-urbain qu'en milieu urbain. Å cet effet, nous formulerons ainsi notre hypothèse :

Les ensembles démographiques restreints $(25000 \mathrm{~h}$. et moins) qui constituent des «milieux humains 》 au sens où les relations y sont personnelles et s'y déroulent sous le signe d'une certaine cohésion (valeurs et normes partagées par exemple) sont le lieu d'une criminalité officielle (statistique) moins importante que celle des grands ensembles métropolitains, au prorata des populations de ces deux types d'ensembles.

Ainsi au prorata de la population, un milieu semi-urbain tel Rivière-du-Loup, devrait être le théâtre d'un moins grand nombre de condamnations pénales que Montréal et cela malgré le fait qu'il existe une plus grande visibilité des citoyens et des déviants en milieu à moindre densité géographique, donc une plus grande facilité à identifier, arrêter et condamner les auteurs des délits et crimes.

\section{Deuxième hypothèse}

Nous posons 1'hypothèse que les interventions des gérants, en vue de contrôler les infractions bancaires, suivent une séquence déterminée allant du moins formel au plus formel. Ainsi, les gérants d'institutions bancaires auraient développé chacun pour soi une gamme de moyens allant du moins formel au plus formel pour rappeler leurs clients à l'ordre. Cette gamme est composée des mêmes éléments d'un gérant à l'autre et présente des sanctions ou des contrôles identiques ou analogues.

\section{Troisième hypothèse}

Nous désirons vérifier si, en milieu semi-urbain, les contrôles dits informels sont proportionnellement plus nombreux qu'en milieu urbain mais moins qu'en milieu rural. A cet effet, nous formulons ainsi notre hypothèse :

Dans les milieux semi-urbains, la déviance et tout particulièrement les délits bancaires donnent lieu à un plus grand nombre de règlements informels que dans les milieux urbains. 
Cette situation (d'informalité) se trouve plus accentuée dans le milieu rural par rapport au milieu semi-urbain.

Ainsi les rappels des gérants de Banques et Caisses populaires à Rivière-du-Loup se limitent plus souvent qu'en milieu urbain (Montréal) et moins souvent qu'en milieu rural (SaintAntonin et Cacouna) à des interventions de type informel.

\section{Quatrième hypothèse}

Nous désirons savoir si l'efficacité de l'intervention du gérant est influencée par une bonne relation gérant-client et comment. L'efficacité se reconnaît au fait que le client, après l'intervention du gérant, en arrive à un arrangement auquel il se tient. Nous posons comme hypothèse que c'est, entre autres choses, la qualité de la relation entre le gérant et son client qui donne son efficacité à l'intervention non formelle du premier. Cette qualité (relation réelle, personnelle et significative) se retrouve plus en milieu semi-urbain qu'en milieu urbain et plus en milieu rural qu'en milieu semi-urbain.

Ainsi les rappels des gérants de Banques et Caisses populaires à Rivière-du-Loup, plus souvent qu'en milieu urbain et moins fréquemment qu'en milieu rural, tout en se limitant à des interventions de type informel (hypothèse 3) réussissent ainsi à élaborer avec les clients, des arrangements auxquels ceux-ci sont fidèles dans la majorité des cas et de plus, réussissent à rappeler le client à l'ordre.

Nous avons rencontré les gérants de huit institutions bancaires et avons cherché à connaître leur "philosophie générale * d'action lors d'irrégularités bancaires (" erreurs » sur les chèques, chèques sans fonds, prêts en retard), leurs méthodes d'interventions auprès des clients, la fréquence de ces interventions, l'efficacité de leurs relations, etc. Les résultats furent les suivants :

\section{A. LA CRIMINALITÉ OFFICIELLE}

À l'appui de notre première hypothèse concernant la criminalité officielle et statistique dans différents milieux, la Statistique de la criminalité et de l'application des règlements de la circulation (Statistique Canada) pour 1972-1973 témoigne du fait que les villes du groupe 1 à 3 (Montréal, Laval, Longueuil et Québec) ont un taux d'infractions (par $1000000 \mathrm{~h}$.) de 7118,4 pour 1973 , alors que les villes du groupe 6 à 9 ( $25000 \mathrm{~h}$. et moins), groupe auquel 
appartient Rivière-du-Loup, ont un taux d'infractions (par 100000 h.) de 4172,7 pour la même année. Il est donc nettement visible, en utilisant un taux comparatif de $100000 \mathrm{~h}$. que les milieux de $25000 \mathrm{~h}$. et moins, milieux qualifiés de semi-urbains, ont un taux d'infractions moins grand que celui des milieux urbains. Le rapport est de 1,7 (urbain) contre 1 (semi-urbain).

Nous avons considéré ces chiffres dans leur ensemble; c'est-à-dire en retenant toutes les infractions et en pondérant avec $100000 \mathrm{~h}$. pour fins de comparaisons. Quittons ces considérations générales sur la criminalité pour insister plus spécifiquement sur la population qui nous intéresse : Rivière-du-Loup. À titre d'exemple, pour la seule année de 1973, les statistiques de police (susmentionnées) font état des chiffres suivants, pour les villes de Montréal et Rivière-du-Loup, à propos des infractions les plus importantes :

\begin{tabular}{lrc}
\hline & Montréal & Rivière-du-Loup \\
\hline Meurtre & 40 & - \\
Tentative de meurtre & 85 & - \\
Homicide involontaire coupable & 3 & - \\
Viol & 114 & - \\
Autres infractions sexuelles & 741 & 54 \\
Blesser & 4541 & $\mathbf{1 7}$ \\
Voies de fait (pas attentat à la pudeur) & 2856 & - \\
Vol qualifié & 18839 & 3 \\
Introduction par effraction & 5962 & 7 \\
Vol véhicule à moteur & 4382 & 26 \\
Vol de plus de $\$ 200$ & 29173 & 85 \\
Vol de moins de $\$ 200$ & 337 & - \\
Possession de biens volés & 3209 & - \\
Fraudes & 415 & - \\
Prostitution & 232 & - \\
Jeux et paris & 301 & - \\
Armes offensives & 741 & - \\
Lois fédérales & 1392 & - \\
Cannabis & 639 & - \\
Lois provinciales & 21 & - \\
Conduite dangereuse & 2569 & 59 \\
Capacité de conduite affaiblie & & \\
\hline
\end{tabular}

Ces chiffres ne sont pas soumis au taux comparatif de $100000 \mathrm{~h}$. La population de Montréal en 1973 étant de $2500000 \mathrm{~h}$. et celle de Rivière-du-Loup (arrondie) de $14000 \mathrm{~h}$., l'on devrait être en mesure d'affirmer un rapport de 175 contre 1 dans le nombre des infractions. Tel n'est pas le cas, loin de là. A titre d'exemple, prenons les vols de plus de $\$ 200$, de moins 
de $\$ 200$, les jeux et paris, ainsi que les fraudes puisque la présente étude porte sur les délits d'ordre économique.

On constate, sauf dans les cas des vols de plus de $\$ 200$ où le ratio est de 1 (en milieu semi-urbain) contre 168 (en milieu urbain) que tous les autres délits retenus sont loin du ratio proportionnel à la population (1 contre 175) auquel on devrait s'attendre. En effet, les vols de moins de $\$ 200$ présentent un rapport de 1 contre 237. Il en est ainsi des chiffres sur les jeux et paris qui représentent un rapport de zéro contre 232. Toutefois, ce n'est pas là l'élément le plus révélateur en ce qui concerne notre sujet précis : le secteur des banques et caisses. Ainsi le taux quasi proportionnel des vols de plus de $\$ 200$ ne se retrouve absolument pas dans les cas de fraudes, secteur qui, parmi ceux énumérés, est le plus proche de notre étude.

En effet, on compte 3209 délits de fraude à Montréal alors que Rivière-du-Loup n'en compte que 8 , ce qui représente un rapport de 401 contre 1 . Nous sommes loin du 175 contre 1 , indiqué précédemment comme proportionnel à la population. Même dans l'ensemble des infractions énumérées précédemment, on remarque, à plusieurs endroits, que certains délits (meurtre, viol, vol qualifié...) ne sont tout simplement jamais commis ou plutôt ne font l'objet d'aucune arrestation à Rivière-du-Loup.

Pour l'ensemble de la criminalité, le ratio proportionnel n'est plus atteint. Ainsi, au prorata de la population, la criminalité officielle est moins grande en milieu semi-urbain qu'en milieu urbain.

\section{B. LA "GAMME » DES MOYENS À LA DISPOSITION DES GÉRANTS}

En ce qui concerne notre deuxième hypothèse traitant de la gamme des moyens qu'auraient développé les gérants pour rappeler leurs clients à l'ordre, nous nous proposons de citer tous les propos pertinents recueillis en entrevue, auprès des gérants. Les propos recueillis portent sur la philosophie générale de chaque gérant en ce qui a trait à ses relations avec ses clients, ainsi que la gamme d'interventions qu'il utilise. Il est important de spécifier que tous les gérants sans exception nous ont signifié clairement que les procédures ou démarches qu'ils entreprenaient pour rappeler un client à l'ordre n'étaient, en aucun cas, inscrites dans un livre ou un code de règlements émanant de leur siège social. 
Tous ont convenu que ces mesures étaient laissées à leur entière discrétion. Ils sont tout de même tenus de rendre compte en haut lieu des résultats de leurs démarches, mais ce sont eux qui décident comment agir avec leurs clients.

Le gérant de la Caisse populaire de Montréal (Ouest) reflète une conception positive et optimiste de l'homme. Il s'exprime ainsi :

Nous croyons que tous (les clients) sont bons jusqu'à ce qu'ils prouvent qu'ils sont mal intentionnés. Lorsque nous ne sommes pas perdants, nous retournons les chèques aux banques (ou bénéficiaires) sans rappeler le client.

Toutefois, plusieurs moyens peu complexes, entrent en jeu dès que la Caisse est perdante (chaque étape suppose que la précédente ne fut pas efficace) :

1. le gérant téléphone au client ;

2. il téléphone au client et l'avise que son chèque est retourné accompagné d'un avis mentionnant la raison du refus ;

3. il envoie une lettre officielle, non une circulaire, recommandée ;

4. il prend une action légale contre son client.

En somme, nous pouvons affirmer que le gérant donne d'abord une "chance » à chaque client en le considérant positivement mais que cette chance est dépassée dès que la caisse est perdante dans l'encaissement du chèque. De plus, dans le cas de cette caisse, il s'agit d'une gamme d'interventions peu complexe par rapport à celle qu'utilisent les autres gérants.

De la même façon, nous avons recueilli toujours en milieu urbain, mais dans le secteur Est, l'opinion d'un gérant, qui explique, à propos des chèques N.S.F. (sans fonds) récoltés à l'épargne : "Nous ne tolérons cela à l'épargne. Nous retournons le chèque le même jour en téléphonant, aucune chance ne lui est donnée. Il ajoute toutefois qu'au secteur commercial, il est plus permissif afin de garder ses clients. Par conséquent, l'on peut dire que ce gérant est strict en ce qui concerne les clients à l'épargne, mais plus large à propos du secteur commercial. D'ailleurs, on voit que l'aspect monétaire compte beaucoup, car financièrement parlant, la perte d'un «client-commercial» a plus de conséquences que la perte d'un "client-épargne ». En ce qui regarde sa gamme d'interventions, le gérant de cette banque de l'Est de Montréal la 
divise en deux, selon qu'elle concerne les effets irréguliers ${ }^{4}$ ou la perception des prêts. Pour les effets irréguliers il procède ainsi :

1. téléphone;

2. retourne le chèque ;

3. tolère un découvert (commercial).

Cependant en ce qui touche le remboursement des prêts, la série de procédés qu'il emploie est plus complexe :

1. téléphone (après 3 jours de retard) à caractère «smooth ;

2 . encore après 3 jours, un avis-circulaire poli, non recommandé, est envoyé ;

3. le directeur appelle personnellement et tente d'obtenir une promesse, c'est aussi sur un ton poli ;

4. une lettre de trente jours (mise en demeure), recommandée ;

5. huissier avec saisie ou arrangement en Cour. La banque n'a connu que deux affaires de cette gravité.

Ce dernier gérant procède donc différemment selon qu'il s'agisse d'un prêt ou d'un effet irrégulier. Nous pouvons remarquer, dans le cas des prêts, que les démarches sont plus complexes mais aussi que le montant de perte (risque) est plus grand que dans les effets irréguliers où les procédures se résument à un avertissement téléphonique. Les deux gammes d'action procèdent, comme on peut s'y attendre, du moins formel (téléphone : verbal) au plus formel (retour du chèque ou huissier). Dans le même secteur (Est) de la ville de Montréal, nous avons retenu aussi la Banque royale "succursale communautaire ». Son caractère spécial (aspect communautaire) se réflète dans la philosophie générale de la direction :

On s'attend à des choses comme celles-là (effets irréguliers).

Ce serait anormal de ne pas en avoir. Donc nous n'avons pas une attitude négative au départ. Il faut chercher s'il y a une «infraction », savoir pourquoi et remonter à la source.

D'ailleurs, sa procédure d'intervention pour rappeler un client à l'ordre est assez détaillée et procède plus dans l'informalité que pour les deux milieux précédents :

1. téléphone (nous retournons quelquefois le chèque le même jour) ;

4. Erreurs plus ou moins < volontaires > sur les chèques tels : signature irrégulière, mauvais folio, mauvaise date, montant écrit différent du montant chiffré... 
2. téléphone ;

3. téléphone ;

4. lettre (non une circulaire), pas sévère, non recommandée ;

5. lettre, non recommandée, pas plus sévère ;

6. lettre recommandée, pas plus sévère ;

7. selon l'importance du montant perdu : poursuites légales.

On remarque ici qu'il y a beaucoup plus d'interventions auprès du client que dans les deux institutions financières précédentes. Cette gamme débute dans l'informalité mais s'achemine plus lentement, avec beaucoup d'étapes, vers un contrôle plus formel.

Nous abordons le secteur semi-urbain, Rivière-du-Loup, en commençant par analyser les données fournies par le gérant de la Banque canadienne nationale. Sa philosophie : "Tout dépend de la bonne foi du client et cela dépend de la fiche du client. On accepte sa parole; en cas de non-respect de sa parole, on ne la croira plus jamais ». En somme ici aussi on est prêt à donner une chance au client, par contre, si le client ne respecte pas sa parole, il sera «banni ». Mais il semble que la promesse du client n'est pas le seul élément de cette philosophie. En effet, on considère aussi la bonne foi du client et ici on entend par bonne foi, la sincérité que le client manifeste par rapport à sa situation financière. La fiche financière du client est aussi considérée. En ce qui concerne les procédures d'interventions de ce gérant, nous remarquons cette échelle :

1. téléphone ;

2. après une journée, on envoie un premier avis écrit ou on retourne le chèque (s'ils ne sont pas perdants). Cet avis écrit est une circulaire non recommandée;

3. après une semaine, on envoie un deuxième avis écrit qui est aussi une circulaire non recommandée ;

4. après une autre semaine, on envoie un troisième avis écrit, qui est aussi une circulaire non recommandée ;

5. lettre recommandée : mise en demeure;

6. si le montant le justifie, on fait appel à un avocat.

Cette démarche est celle employée pour le recouvrement des prêts. En ce qui concerne les effets irréguliers, les démarches sont moins strictes et n'atteignent pas un tel degré de complexité. Il n'y a pas de circulaire (avis) pour les effets irréguliers alors que 
dans les cas de prêts, l'envoi de ces polycopies est automatique. En somme, cette démarche s'apparente à celle des milieux urbains, sauf que la majorité des écrits ne sont qu'une formalité (automatique) à laquelle le gérant acquiesce et qu'il insère parmi ses démarches personnelles tels téléphones, lettres recommandées... La deuxième institution financière de Rivière-du-Loup a comme philosophie générale : la communication et l'explication : « Nous contactons nos clients pour qu'ils fassent un dépôt dans la journée même ou qu'ils fournissent une explication satisfaisante à courte échéance ». Ici on semble plus ouvert au dialogue. On accepte même un bref retard dans le cas où la faute est e justifiée ». A cette banque on procède de la façon suivante pour recouvrer les prêts :

1. téléphone;

2. rencontre ;

3. premier avis écrit, peu sévère, non recommandé ;

4. deuxième avis écrit, plus sévère, non recommandé ;

5. mise en demeure, recommandée ;

6. avocat.

On remarque, au premier coup d'œil, un élément nouveau : la rencontre. Peu de gérants nous ont manifesté cette intention de rencontrer le client pris en défaut. Cette rencontre est amicale et a pour but de connaître les raisons du retard du versement et au besoin d'établir un échéancier différent et plus adapté au client. C'est aussi l'une des premières fois (sauf à la Banque royale à caractère communautaire) que l'on rencontre plus d'une démarche informelle pour la perception des remboursements. La gamme se trouve simplifiée lorsqu'il s'agit d'effets irréguliers :

1. téléphone :

2. téléphone (rappel) ;

3. retourne le chèque car \& nous n'encaissons pas les chèques douteux, ainsi nous sommes rarement perdants ».

Ici aussi, la communication verbale est plus fréquente qu'à la Caisse de Montréal (Ouest) et à la Banque provinciale de Montréal (Est). En effet, à la Caisse, le deuxième téléphone avait pour but d'aviser le client que son chèque lui avait été retourné, alors qu'à la Banque provinciale (Est) on ne note qu'un appel. Donc, en milieu semi-urbain il y a deux démarches informelles avant de retourner le chèque en défaut. Finalement, ici aussi le 
gérant agit selon une gamme de contrôles allant du moins formel au plus formel. Toutefois, l'informalité en question comporte plus qu'une communication verbale, il y a aussi une rencontre de personne à personne.

La dernière institution financière étudiée, en milieu semiurbain, est une Caisse populaire. La philosophie générale du gérant consiste à "prendre des arrangements téléphoniques avec les individus concernés \$. Elle est donc ouverte à la discussion et en aucun moment nous n'avons perçu que le client n'aurait qu'une seule chance, d'ailleurs, la discussion se poursuit jusqu'à une entente ou un arrangement. Ce gérant explique qu'il a sensiblement les mêmes démarches pour la perception des prêts que pour les effets irréguliers :

1. téléphone :

2. téléphone ;

3. lettre amicale, non recommandée, ce n'est pas une circulaire ;

4. lettre plus sévère, recommandée (mise en demeure) ;

5. avocat.

Lorsque nous avons demandé au gérant si sa lettre n'était pas une circulaire, il s'est exclamé négativement, en affirmant qu'il possédait de telles circulaires mais qu'il ne s'en servait presque jamais. Il préfère écrire une lettre personnelle à chaque client. Nous voyons donc ici que, même dans les cas de formalités, ce gérant préfère personnaliser ses démarches, ce qui n'avait pas été le cas ailleurs. Même la teneur de la première lettre est (dans ses propres mots) amicale.

Le dernier milieu étudié, rural, comprend deux villages. La gérante de la Caisse populaire de Saint-Antonin explique ainsi sa philosophie générale : «Nous sommes d'abord tolérants, par la suite nous usons d'un avertissement sérieux et finalement, nous retournons le chèque ». Déjà on voit qu'il y aura deux étapes avant le renvoi d'un chèque. On commence par communiquer avec le client, on lui donnera plus d'une chance. Pour la perception des prêts et les effets irréguliers, le gérant procède de la même manière, soit :

1. téléphone :

2. retourne le chèque avec $\$ 2$ de frais d'administration;

3. un avis-circulaire recommandé ; 
4. il ferme le compte. Cela n'est arrivé que deux fois en douze ans. D'ailleurs, aussi dans le cas des prêts, il n'y a que deux cas de retard de versement en douze ans.

Devant notre étonnement vis-à-vis le peu de démarches formelles effectuées, la gérante nous explique qu'un second téléphone n'est même pas nécessaire (pas plus que de retourner le chèque ou d'envoyer un avis) car elle estime que $98 \%$ de ces cas se règlent dès la première communication téléphonique. Cependant, même si les «échelons» formels de la gamme de moyens ne servent que très rarement, nous pouvons tout de même observer que cette gérante a une gamme d'interventions peu complexe mais tout aussi efficace sinon plus que les précédentes, allant du moins formel au plus formel.

Pour ce qui est de la Caisse populaire de Cacouna, on y retrouve une philosophie des plus larges :

A la première, deuxième, troisième ou quatrième fois on garde le chèque jusqu'au lendemain. Si l'individu n'est pas venu, on peut lui téléphoner et l'avertir, ou lui retourner le chèque. Nous procédons verbalement plutôt que par écrit.

Ici aussi la tolérance ne se limite pas à une chance, mais à plusieurs concessions. En ce qui concerne le recouvrement des prêts et le règlement des effets irréguliers, le gérant procède de la même manière :

1. un premier coup de téléphone amical ;

2. un téléphone plus direct;

3. un téléphone sévère avec menace de retourner le chèque ;

4. on retourne le chèque sans téléphoner, avec un aviscirculaire ;

5. on demande au conseil d'administration l'autorisation de fermer le compte.

On remarque que sa gamme d'interventions comprend beaucoup d'échelons informels. Nous croyons utile de citer le passage suivant afin de montrer la teneur de cette gamme et son efficacité :

En trois ans, j'ai été une fois en recouvrement (avocat) pour un prêt personnel trop retardé. Présentement, nous avons peut-être dix cas sur deux cents qui ont un léger retard, mais ils ne nous causent pas d'inquiétude. De plus, nous avons pour un million de prêts hypothécaires et pas un seul n'a eu un mois de retard. 
Il s'avère donc que cette gamme de moyens, allant du moins formel au plus formel et se situant surtout dans l'informalité, est des plus efficaces. Pour terminer l'analyse des résultats fournis par les réponses des gérants concernant la deuxième hypothèse, nous utilisons un tableau récapitulatif des principaux éléments recueillis dans chaque milieu.

\begin{tabular}{|c|c|c|c|}
\hline $\begin{array}{c}\underset{\text { Milien }}{\text { informalité }} \\
\text { formalité }\end{array}$ & Urbain & Semi-urbain & Rural \\
\hline \begin{tabular}{c|} 
Formel \\
$\uparrow$
\end{tabular} & 1. téléphone & $\begin{array}{l}\text { 1. téléphone } \\
\text { 2. téléphone } \\
\text { (rencontre) }\end{array}$ & $\begin{array}{l}\text { 1. téléphone } \\
\text { 2. téléphone } \\
\text { 3. téléphone }\end{array}$ \\
\hline$\underset{\text { Informel }}{\downarrow}$ & $\begin{array}{l}\text { 2. circulaire et } \\
\text { retour chèque } \\
\text { 3. lettres recom- } \\
\text { mandées } \\
\text { 4. Cour }\end{array}$ & $\begin{array}{l}\text { 3. circulaire ou } \\
\text { retour chèque } \\
\text { 4. lettre recom- } \\
\text { mandée } \\
\text { 5. Cour }\end{array}$ & $\begin{array}{l}\text { 4. retour chèque } \\
\text { ou circulaire } \\
\text { 5. fermer le } \\
\text { compte }\end{array}$ \\
\hline
\end{tabular}

Il est à remarquer que ce tableau ne contient que la nomenclature des "éléments » de cette gamme. Nous n'avons pas donné le poids ou le nombre des interventions à chaque stade. Nous verrons ultérieurement que $75 \%$ à $95 \%$ des interventions de tous les gérants s'avèrent efficaces dès le niveau informel. Les étapes formelles sont donc plus rarement employées.

\section{FRÉQUENCE D'UTILISATION DES MOYENS INFOR- MELS}

En ce qui concerne la troisième hypothèse qui porte sur l'utilisation plus fréquente des moyens informels par rapport aux moyens formels en milieu rural et inversement en milieu urbain, l'on pourrait en trouver une confirmation (assez fragile, il est vrai, si on la soumet à la rigueur d'une analyse mathématique) dans le fait que les villes du groupe 6 à $9(25999 \mathrm{~h}$. et moins) tendent à classer sans mise en accusation une plus forte proportion d'affaires criminelles que les grandes villes du groupe 1 à $3(13,4 \%$ d'affaires non suivies de mise en accusation dans les premières, contre $8,7 \%$ dans les secondes) et cela, malgré le fait que les villes petites et moyennes réussissent $a$ classer un plus fort pourcentage de leurs affaires criminelles que les grandes villes (Statistiques Canada, 1974, p.5.2.). De nos entrevues, l'on peut tirer des 
conclusions beaucoup plus fortes à l'appui de cette hypothèse, comme on le voit au tableau qui suit, les pourcentages (avoués) de règlements informels des affaires bancaires diminuent avec la densité démographique, ou si l'on préfère, la formalité est vraiment plus caractéristique des contrôles exercés par les gérants urbains $(18 \%)$ que ruraux (1\%) pour ce qui est des institutions étudiées. Il va de soi que ces proportions indiquent des tendances plutôt que des faits bien mathématiquement contrôlés, car il s'agit ici des estimations des gérants.

Ce tableau s'interprète de la façon suivante. On trouve dans la première colonne la liste des milieux sur lesquels a porté l'étude et le nom de chaque institution visitée. La deuxième colonne indique le nombre de clients ou sociétaires que compte chaque institution. Cette colonne permet de nous donner une idée de l'importance relative de l'institution bancaire. La troisième colonne indique le nombre d'interventions qu'un gérant doit faire, en moyenne, par jour; ces interventions sont uniquement des rappels à l'ordre (contrôles). Dans la quatrième colonne, figurent les taux dont l'indice permettra de comparer le pourcentage moyen des démarches journalières d'un gérant par rapport à sa clientèle. Ce taux est obtenu ainsi :

nombre d'interventions par jour

nombre de clients.

Dans la cinquième colonne, on trouve le pourcentage approximatif des interventions de chaque gérant considérées comme informelles (selon le tableau récapitulatif précédent où ici chaque gérant a évalué en \% chacun de ses types d'interventions). La sixième colonne est conséquente à la précédente. Une moyenne permet de comparer selon le milieu. A titre d'exemple, la première ligne du tableau se lirait et s'interpréterait comme suit : dans l'Ouest du milieu urbain, nous avons fait enquête auprès du gérant d'une Caisse populaire, Caisse qui comprend 6500 sociétaires. Le gérant effectue en moyenne 25 « rappels à l'ordre * en une journée. Son taux d'activité (de contrôle) est de $0,4 \%$, c'est-à-dire que moins de $1 \%$ de sa clientèle fait l'objet de « rappels » en une journée. Soixante-quinze pour cent de ses interventions sont d'ordre informel, les autres ( $25 \%$ ) étant dites formelles. En comparaison avec son confrère du secteur Est de Montréal, il consacre beaucoup moins de temps à "contrôler > ses clients (taux de $0,4 \%$ comparativement à $1 \%$ ). 
PLACE (\%) DES INTERVENTIONS INFORMELLES

DANS L'ENSEMBLE DES INTERVENTIONS DES GÉRANTS

\begin{tabular}{|c|c|c|c|c|c|c|}
\hline & Institutions & $\begin{array}{l}\text { Nombre de } \\
\text { clients }\end{array}$ & $\begin{array}{l}\text { Nombre } \\
\text { d'interventions } \\
\text { (jour) }\end{array}$ & $\%$ & $\begin{array}{l}\text { \% approximatif } \\
\text { d'interventions } \\
\text { informelles }\end{array}$ & $\begin{array}{l}\text { \% approximatif } \\
\text { d'interventions } \\
\text { formelles }\end{array}$ \\
\hline \multirow[t]{2}{*}{ 昜 } & $\begin{array}{l}\text { Ouest : Caisse populaire } \\
\text { Est : Banque provinciale } \\
\\
\end{array}$ & $\begin{array}{l}6500 \\
3100 \\
2500\end{array}$ & $\begin{array}{l}25 \\
30 \\
5 \text { à } 6\end{array}$ & $\begin{array}{l}0,4 \% \\
1,0 \% \\
0,2 \%\end{array}$ & $\begin{array}{l}75 \% \\
80 \% \\
90 \%\end{array}$ & $\begin{array}{l}25 \% \\
20 \% \\
10 \%\end{array}$ \\
\hline & Moyenne : & - & - & $\longrightarrow$ & $82 \%$ & $18 \%$ \\
\hline \multirow[t]{2}{*}{ 亶量 } & $\begin{array}{l}\text { Banque canadienne nationale } \\
\text { Banque provinciale } \\
\text { Caisse populaire }\end{array}$ & $\begin{array}{l}3000 \\
1775 \\
4500\end{array}$ & 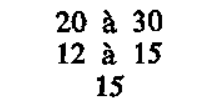 & $\begin{array}{l}0,8 \% \\
0,8 \% \\
0,3 \%\end{array}$ & $\begin{array}{l}85 \% \\
90 \% \\
85 \%\end{array}$ & $\begin{array}{l}15 \% \\
10 \% \\
15 \%\end{array}$ \\
\hline & Moyenne : & $\longrightarrow$ & - & 一 & $87 \%$ & $13 \%$ \\
\hline \multirow[t]{2}{*}{ 䑐 } & $\begin{array}{l}\text { Caisse Saint-Antonin } \\
\text { Caisse Cacouna }\end{array}$ & $\begin{array}{l}1500 \\
1275\end{array}$ & $\begin{array}{l}2 \\
5\end{array}$ & $\begin{array}{l}0,1 \% \\
0,4 \%\end{array}$ & $\begin{array}{l}98 \% \\
99 \%\end{array}$ & $2 \%$ \\
\hline & Moyenne : & - & - & - & $99 \%$ & $1 \%$ \\
\hline
\end{tabular}


Maintenant que nous avons abordé, à titre d'exemple seulement, l'étude d'un milieu, nous pouvons comparer les trois milieux l'un à l'autre par l'indice de formalité des interventions ; nous considérons surtout les interventions informelles car ce sont elles qui représentent l'objet principal de cette étude. Ces approximations fournies par les gérants même, révèlent que le milieu urbain a le moins fort pourcentage d'interventions informelles $(82 \%)$, que le milieu semi-urbain emploie plus de contrôles informels $(87 \%)$ que le milieu urbain, mais moins que le milieu rural $(99 \%)$ qui utilise presque uniquement des contrôles dits informels. Ainsi, dans le milieu semi-urbain, les délits bancaires donnent lieu à un plus grand nombre de contrôles et de règlements informels que dans les milieux urbains. Cette situation (d'informalité) se retrouve en plus accentuée en milieu rural par rapport au milieu semi-urbain.

\section{L'EFFICACITÉ DU CONTRÔLE INFORMEL}

À l'appui de la quatrième hypothèse concernant l'efficacité du contrôle informel du gérant, nous verrons pour chacun : 1) ce qui, selon lui, établit la qualité de la relation entre le gérant et son client ; 2) ce qu'il pense être le fondement de l'efficacité de son intervention; 3) le poids relatif de quelques variables (sexe, âge, compte en banque, renommée financière et sociale) susceptibles d'influencer la relation entre le gérant et un client. Dans le secteur Ouest du milieu urbain, le gérant de la Caisse populaire décrit ainsi les «bonnes» et «mauvaises » relations entre lui et son client :

Les relations commencent toujours par être bonnes. Il en va de cela comme de notre philosophie : toute personne est considérée comme bonne tant qu'on n'a pas eu la preuve du contraire. C'est seulement à ce moment (lorsqu'il y a eu faute) qu'on peut parler de mauvaises relations gérant-client.

On doit donc considérer qu'à défaut de preuve du contraire, le client est considéré comme solvable par le gérant. Au fond, les clients sont inconnus du gérant jusqu'à ce que survienne une difficulté. Ainsi, ceux que le gérant connaîtra de ses 6500 clients, éparpillés parmi près de 34000 personnes qui composent le secteur géographique, seront ceux : 1) avec qui il a eu à communiquer (par téléphone ou par écrit) pour des effets irréguliers ou autres ;2) ceux qui l'ont rencontré pour d'autres raisons que nous 
n'avons pas cherchées à connaître. À l'appui de cette affirmation le gérant explique :

... on connaît quelque peu nos personnes par téléphone. De plus, ce sont généralement les mêmes qui ont des effets irréguliers, ce qui implique une relation. Ces erreurs sont plus ou moins « volontaires » et généralement, elles sont faites dans le but de bénéficier d'un court délai.

Le chèque étant retourné d'une Caisse à l'autre avec les mentions du refus (N.S.F., signature irrégulière...), il n'est encaissé qu'avec un retard de deux à dix jours. Ce retard suffit parfois au signataire pour lui permettre de trouver un montant capable de compenser pour ce « découvert». Lorsque nous avons cherché à connaître les variables (citées précédemment) qui pouvaient l'influencer lors de ses relations avec ses clients, nous avons constaté que seule la « régularité » dans le compte du client était retenue. "Ces points (variables) ne sont pas considérés *. Nous nous contentons de la régularité dans le compte. Dans l'ensemble, il en ressort que le gérant connaît très peu ses clients car il en a trop, mais, c'est justement une caractéristique propre au milieu urbain (anonymat). Étant donné qu'il ne connaît pratiquement que ses clients "fautifs », et certains autres qui présentent des demandes exceptionnelles, et que même à l'endroit de ce groupe restreint, il emploie souvent comme mode de contact le téléphone, nous ne pouvons guère parler de relations réelles, personnelles et significatives entre ce gérant et ses clients.

Toujours en milieu urbain, mais dans le secteur Est, nous avons deux banques dont l'une à statut spécial (succursale communautaire). Nous les analysons ensemble pour mieux les différencier. En ce qui concerne la nature de la relation gérant-client à la Banque provinciale : "Nous cherchons : 1) à connaître l'état financier du client ;2) à amener le client à administrer son compte. Le gérant n'a pas toujours à communiquer avec lui à cause de chèques N.S.F. Cependant, le client doit toujours répondre lorsqu'on l'appelle, c.-à-d. : il en vient à un arrangement qu'il maintiendra. » $\grave{A}$ la Banque royale, succursale communautaire, le gérant donne une réponse courte et précise : "C'est le respect mutuel des deux », qui caractérise la relation gérant-client. La différence est sensible : pour le premier, la relation gérant-client se limite à des obligations qui, notons-le, incombent au seul client. Rien ici d'une relation personnelle et significative : 1) la relation est à sens unique : client au gérant ; 2) la relation est contrai- 
gnante pour le client seulement. À l'opposé, le gérant de la Banque royale communautaire : 1) n'a aucune restriction financière ou quantitative ; 2) sa relation qualitative est à deux sens : elle implique le respect mutuel des parties.

Lorsque nous avons vérifié si c'est la qualité de cette relation qui assure l'efficacité de leurs interventions informelles, les deux gérants ont répondu affirmativement, celui de la Banque provinciale ajoutant toutefois ce commentaire : "ainsi on règle plus de problèmes par téléphone que par courrier $\gg$. Il appert donc que l'informalité dans ses relations donnent de meilleurs résultats que la formalité. Ce fait vaut pour tous les milieux, mais avec des nuances. Ainsi, il est plus prononcé dans le milieu semi-urbain qu'urbain et encore plus en rural qu'en semi-urbain. On perçoit aussi que, si le gérant de la Banque provinciale préfère les contrôles informels aux formels, l'intérêt d'une relation plus humaine ou plus chaleureuse n'est pas en cause. C'est le rendement seul qui est considéré : "ainsi, on règle plus de problèmes ». Pour ce même gérant, l'âge et le sexe des clients importent peu dans ce genre de relations, mais il remarque : "nous avons plus de troubles avec les 18 à 20 ans et les 30 à 50 ans ». C'est dire que les difficultés ne manquent pas puisque les «sages », soit les 20 à 30 ans et les plus de 50 ans ne représentent qu'une proportion de sa clientèle. Selon lui : «la renommée sociale importe peu ». Ce qui le détermine c'est le montant en Banque, la réputation financière ainsi que la race; il est le seul à avoir retenu cette dernière variable. Il semble que son expérience personnelle fut des plus mauvaises avec les Noirs et les immigrants.

Ainsi donc les variables retenues par ce gérant (compte bancaire, race) se situent loin d'une relation personnelle. Alors que l'inverse se produit chez le gérant de la Banque royale communautaire pour qui ni l'âge, ni le sexe, ni le montant en banque ne sont en soi déterminants. C'est le respect mutuel qui motive l'action. À la base de cette relation : la franchise. Les variables retenues sont donc qualitatives.

En milieu semi-urbain, la qualité de la relation est établie à partir de certains points qui varient d'une banque à l'autre. À la BCN on dira, en parlant des clients : « C'est l'image de sa carte ", soit sa fidélité à respecter ses engagements en cas d'emprunts. À la Banque provinciale, c'est : «la confiance » avec, en plus, la nécessité pour la banque «de bien conseiller le client». 
À la Caisse populaire : "L'accueil compte pour beaucoup. On doit mettre le client en confiance. On lui montre qu'on a tout notre temps à lui consacrer, il faut lui faire sentir qu'on est là pour l'aider. \$ De ces déclarations on peut en déduire qu'à la $\mathrm{BCN}$, on se fie davantage à la réputation financière (quantitatif), alors que la Banque provinciale et la Caisse populaire mettent le point sur la confiance et admettent des devoirs de la part du gérant : qui doit donner des conseils qualifiés et le temps voulu. Voilà des éléments qualitatifs propres à faciliter l'établissement de rapports mutuels plus significatifs et humains. Le gérant de la $\mathrm{BCN}$ élabore sur l'efficacité de l'intervention informelle :

... il y a même beaucoup de clients qui, suite au premier coup de téléphone, demandent de ne pas envoyer d'avis écrit (pour que leur entourage ne soit pas au courant du découvert) et qu'ils viendront régler cela dans la journée.

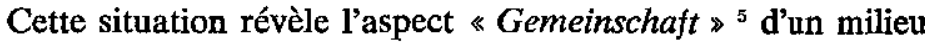
semi-urbain où l'entourage est susceptible de certaines réactions s'il est informé d'irrégularités bancaires commises par son voisin. La pression sociale (contrôle de la société ou du groupe) est évidente et si forte ici que la simple menace d'un avis écrit suffit, dans la majorité des cas, à rappeler le client à l'ordre.

À la Banque provinciale, c'est «par la confiance » que le gérant explique l'efficacité de son intervention informelle. Le gérant de la Caisse populaire est aussi du même avis. En plus, ce dernier est le seul à avoir parlé de ses interventions formelles : «... mais je vous dis que pour les $25 \%$ ou $15 \%$ autres, on est plus direct et moins accueillant ».

À l'intérieur de ces politiques de base, on tient cependant compte d'autres données. À la BCN : «Un bon client s'apparente à l'image de sa carte. Tout compte, par exemple, l'âge, s'il n'a que 18 ans, nous serons plus sévères et nous retournerons le chèque la journée même ou enverrons l'écrit, » Pour la Banque provinciale, le système est plus complexe : "Si c'est un jeune, je tiens compte : a) de sa valeur morale, c.-à-d. : si je connais le père (réputation ou la famille), b) s'il a un emploi stable, c) l'administration de son compte si petit soit-il. S'il a un certain âge (32-35 ans, marié, père) je tiens compte : a) des antécédents

5. La Gemeinschaft , est entendue ici au sens où Tonnies F. (Gemeinschaft und Gesellschaft, Michigan, 1957) l'emploie. Il s'agit d'une - Communauté ", par opposition à la "Société ", où les rapports sont réels, personnels et significatifs. La famille en est l'exemple typique. 
financiers par les bureaux de crédits, b) de sa stabilité d'emploi. , Nous retrouvons ici encore le contexte de \&Gemeinschaft \$ où la réputation de la famille et la valeur morale jouent le rôle de garants. Finalement, à la Caisse populaire, on retenait : « le montant en dépôt [...] ainsi que la régularité des paiements ».

C'est le temps de remarquer qu'un régime financier ne peut être perçu sans considérer le contexte social dans lequel il opère. Ce qui fait ici la différence d'un milieu social à l'autre, c'est qu'en milieu urbain, on utilise presque uniquement des critères quantitatifs (compte en banque, régularité dans paiements), alors qu'en milieu semi-urbain on utilise et des critères quantitatifs et des critères qualitatifs.

En milieu rural, notamment à Saint-Antonin, la relation gérant-client repose sur l'obligeance et l'accueil faits au client : "ça dépend comment on reçoit le client et on peut l'aider à planifier ses affaires $\gg$ et aussi... on offre nos services, il sent qu'on

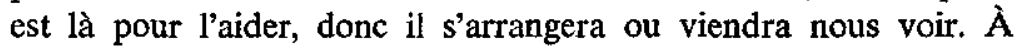
Cacouna on nous dira : «... seules la franchise et la sincérité comptent. En aucun moment, c'est le montant d'argent ». Ici, l'on se défend même de tenir compte de l'aspect quantitatif ou financier. Dans les deux villages, on parle de relations dans les deux sens et les termes sont qualitatifs (offrir ses services, confiance mutuelle). Il s'agit donc de rapports ayant un contenu humain certain. A Saint-Antonin, on nous dit encore :

... l'âge compte. Un jeune sera plus surveillé, plus suivi. Le sexe n'importe pas, la renommée sociale non plus : que le curé fasse un chèque N.S.F., il $\mathrm{y}$ aura $\$ 0.50$ à payer aussi, c'est la même chose pour quelqu'un qui serait sur la Loi Lacombe.

Notons ici le besoin de souligner l'uniformité du traitement en cas d'infraction ou de sanction. À Cacouna, nous entendons une déclaration à peu près similaire :

... non, rien de cela compte (âge, sexe, renommée financière...) sauf la gravité de l'infraction et c'est rarement grave ici. Nous n'avons eu qu'un cas de kiting ${ }^{6}$ et c'était un de mes copains intimes. J'ai fait régler cela par le gérant de l'autre banque impliquée. Ça ne s'est jamais reproduit.

6. Le kiting est une fraude bancaire majeure qui implique des chèques sans fonds dans trois banques différentes. Très difficile à mettre à jour, c'est le troisième gérant qui, découvrant la fraude le dernier, se verra victime de la fraude. 
On voit donc que dans ce milieu rural, l'aspect «Gemeinschaft » est encore plus fort qu'en milieu semi-urbain, puisqu'il fut difficile, sinon impossible, au gérant de régler lui-même ce problème bancaire. Les relations personnelles dans une * Gemeinschaft » sont plus fortes et les relations d'affaires peuvent entrer en conflit plus souvent avec des clients connus intimement. Finalement, nous pouvons faire le lien entre les hypothèses deux et trois, en rappelant que les gérants des milieux ruraux possèdent aussi une gamme de contrôles allant du moins au plus formel et que, dans près de $99 \%$ des cas, on a réglé les difficultés en utilisant des moyens informels. Ainsi, les gérants des institutions bancaires rurales, tout en se limitant à des contrôles informels, réussissent à élaborer avec leurs clients des arrangements auxquels ceux-ci sont, en général, fidèles et à faire rentrer leurs clients dans l'ordre, en cas d'irrégularités.

Nous croyons avoir démontré certains aspects particulièrement «humains de la vie en milieu semi-urbain et en milieu rural. En comparant à ces milieux le « cas exceptionnel », soit la Banque royale communautaire, nous constatons que celle-ci a réussi quelque chose de particulier de par son statut spécial. Son gérant a établi avec ses clients des relations analogues à celles décrites en milieu rural, donc plus informelles et aussi plus humaines que celles développées en milieu semi-urbain et surtout en milieu urbain, milieu dans lequel il est pourtant installé. Cette succursale communautaire nous a permis de voir que la variable milieu n'est pas la seule à pouvoir inévitablement influencer la qualité des contrôles. On dit, il est vrai, de ce quartier HochelagaMaisonneuve qu'il est spécial, car c'est un "village dans la ville *. C'est vrai, mais il n'est pas le seul. Son caractère marquant est surtout d'être défavorisé par contraste avec le district Côte-desNeiges qui est aussi un «village dans la ville, , mais un village favorisé, résidentiel et intellectuel. Ne pourrait-on découvrir un aspect plus humain, plus communautaire, plus «Gemeinschaft 》 à ces quartiers défavorisés ? N'est-ce pas dans les ghettos que la vie de groupe est la plus forte ? Voilà peut-être des éléments qu'une autre recherche pourrait cerner.

Pour résumer, nous ne nions pas l'influence de la variable environnement, mais nous ne pouvons ignorer l'influence de la variable gérant sur la résolution des infractions bancaires. En 
somme, l'explication de la résolution informelle de la majeure partie des infractions bancaires nous viendrait de l'analyse multivariée, car elle répond à de multiples facteurs. Rappelons les constatations de cette étude, soit :

a) notre milieu semi-urbain est le lieu d'une criminalité statistique officielle moins grande que dans les grands ensembles métropolitains ;

b) les gérants des trois milieux possèdent une gamme d'interventions personnelles pour rappeler leurs clients à l'ordre et cette gamme va des moyens informels aux moyens les plus formels ;

c) dans les milieux ruraux, on utilise presque uniquement des moyens de contrôles informels dont l'efficacité est accrue grâce à la qualité des relations gérants et clients et à l'élément «Gemeinschaft \$;

d) la même situation se répète en milieu semi-urbain, mais en moindre proportion qu'en milieu rural ;

$e$ ) en milieu urbain, où pourtant les relations gérant-client sont dénuées de tout caractère intime, on utilise quand même à $80 \%$ ces contrôles informels ;

f) ces contrôles informels ont la préférence, en milieu urbain, pour de strictes raisons d'efficacité puisqu'ils prennent moins de temps et donnent tout de même des résultats appréciables.

$Y$ aurait-il moyen, en milieu urbain, de rendre plus efficaces, non seulement en terme de rendement financier, mais en termes de valeurs humanitaires, ces contrôles informels des gérants de banques? N'est-il pas plus facile d'influencer ces gérants que toute une société ? Nous connaissons au moins un gérant en milieu urbain qui, lui, a tenté et réussi cette chose difficile qui consiste à faire d'un établissement financier, situé en milieu défavorisé, un milieu urbain, non anonyme.

En conclusion, on peut affirmer que si une aussi grande proportion de délits sont réglés informellement, sans l'aide de l'appareil officiel de la justice (la Cour), par le moyen de la conciliation c'est parce qu'il s'avère que cette méthode est plus profitable au banquier, au délinquant, à la victime et qu'elle sert en même temps les prérogatives et les intérêts de la société. 
De nos jours, notre droit (organe formel) s'oriente vers la déjudiciarisation ${ }^{7}$ de certains délits. Ne pourrait-on pas appliquer dans d'autres secteurs de l'action judiciaire ce que nous avons appris de l'expérience efficace, humanitaire et informelle des gérants de banques et de Caisses populaires ? Les conséquences de telles applications pourraient être intéressantes à envisager. Ainsi nous pourrions voir disparaître ou s'atténuer les méfaits de l'étiquetage et de la stigmatisation dans certains délits mineurs. Avant même l'intervention de la Cour, la victime pourrait être indemnisée tel que recommandé aujourd'hui dans notre code criminel. Grâce à cette intervention informelle, le « délinquant » se sentirait moins écrasé que par la sentence habituelle imposée par la « société vengeresse \ qui représente l'intervention formelle. Ainsi, dans certains secteurs de l'activité judiciaire, des règlements informels pourraient mieux servir les intérêts des personnes impliquées qu'un contrôle formel (marquant).

7. A cet effet, voir : * Commission de réforme du droit du Canada , la Déjudiciarisation, document de travail, no 7 , Ottawa, Information Canada, 1975. 